\title{
SHORT-TIME EXISTENCE FOR THE SECOND ORDER RENORMALIZATION GROUP FLOW IN GENERAL DIMENSIONS
}

\author{
KARSTEN GIMRE, CHRISTINE GUENTHER, AND JAMES ISENBERG
}

(Communicated by Lei $\mathrm{Ni}$ )

\begin{abstract}
We prove local existence for the second order Renormalization Group flow initial value problem on closed Riemannian manifolds $(M, g)$ in general dimensions, for initial metrics whose sectional curvatures $K_{\pi}$ satisfy the condition $1+\alpha K_{\pi}>0$, at all points $p \in M$ and for all planes $\pi \subset T_{p} M$. This extends results previously proven for two and three dimensions.
\end{abstract}

The second order approximation of the Renormalization Group flow for the nonlinear sigma model of quantum field theory, which we label the RG-2 flow, is specified by the PDE system

$$
\frac{\partial}{\partial t} g=-2 \mathrm{Rc}-\frac{\alpha}{2} \mathrm{Rm}^{2}
$$

Here $g$ is a Riemannian metric, Rc is its Ricci curvature, $\mathrm{Rm}_{i j}^{2}=g^{p k} g^{q l} g^{n m} R_{i k l m}$ $R_{j p q n}$, and $\alpha$ is a positive parameter. We note that for our purposes here, $\alpha$ can assume any real value. For $\alpha=0$, this system (1) reduces to the Ricci flow. One can see that the sign of the right hand side, which is roughly $1+\alpha \times$ Curvature, should have an impact on the behavior of the flow, and this has been confirmed in various settings: in particular, the size of the term influences the parabolicity of the flow. Oliynyk has shown in [10] that on a two-dimensional manifold, if the Gaussian curvature $K$ satisfies the condition $1+\alpha K>0$, then the flow is (weakly) parabolic; while if $1+\alpha K<0$, then the flow is backward parabolic. In [3], Cremaschi and Mantegazza prove that short-time existence holds in three dimensions so long as the analogous curvature condition $1+\alpha K_{\pi}>0$ is satisfied for all sectional curvatures $K_{\pi}$. In this note we extend this curvature criterion for short-time existence for RG-2 flow to all dimensions, as first announced in [7]. Our main result is the following:

Theorem 1. Let $\left(M, g_{0}\right)$ be a closed $n$-dimensional Riemannian manifold. If $1+$ $\alpha K_{\pi}>0$ for all sectional curvatures $K_{\pi}\left(g_{0}\right)$, at all points $p \in M$ and planes

Received by the editors January 7, 2014.

2010 Mathematics Subject Classification. Primary 53C44.

The first author was partially supported by the NSF under grant DGE-1144155.

The second author was partially supported by the Simons Foundation Collaboration Grant for Mathematicians - 283083.

The third author was partially supported by the NSF under grant PHY-1306441 at the University of Oregon. He also wishes to thank the Mathematical Sciences Research Institute in Berkeley, California, for support under grant 0932078 000. Some of this work was carried out while the third author was in residence at MSRI during the fall of 2013. 
$\pi \subset T_{p} M$, then there exists a unique solution $g(t)$ of the initial value problem $\frac{\partial}{\partial t} g=-2 \mathrm{Rc}-\frac{\alpha}{2} \mathrm{Rm}^{2}, g(0)=g_{0}$, on some time interval $[0, T)$.

Remark 2. In [10, Oliynyk finds open subspaces of the space of smooth metrics that are invariant under the two-dimensional RG-2 flow, and for which the flow remains parabolic (resp. backward parabolic). We are currently investigating this for general dimensions.

Proof. To prove the theorem, we calculate the principal symbol of the DeTurckmodified version of RG-2 flow, which is generated by the PDE system (compare with (10) above)

$$
\frac{\partial}{\partial t} g_{i j}=-2 R_{i j}+L_{W_{u, g}} g_{i j}-\frac{\alpha}{2} \mathrm{Rm}_{i j}^{2} .
$$

Here $W_{u, g}=-g^{i j} u_{j k}^{-1} g^{k l} g^{p q}\left(\nabla_{p} u_{q l}-\frac{1}{2} \nabla_{l} u_{p q}\right)$ is the standard vector field usually chosen to modify the Ricci flow into the related (parabolic) DeTurck version of Ricci flow, with $u$ a fixed metric. Letting $\varphi_{t}$ be the one-parameter family of diffeomorphisms generated by the vector field $-W_{u, g}$, then $\varphi_{t}^{*} g$ is a solution of the RG-2 flow (see also [8). As in the analogous Ricci flow case, if one can show (for a class of choices of the initial metric) that the PDE system (2) is parabolic, then short-time existence holds for the RG-2 flow (1) as well as for the DeTurck-modified flow (2).

To calculate the symbol of the system (2), we first linearize the flow. For the first two terms on the right hand side of (2), this linearization effectively produces the Laplacian (see 4], or Theorem 2.1 in [5]). For the remaining term, $\mathrm{Rm}^{2}$, it is useful to recall the formula for the variation of the Riemann curvature tensor with respect to the metric (see pg. 74 in [2]):

$$
\begin{aligned}
{\left[D \operatorname{Rm}_{g}(h)\right]_{i j k}^{l}=} & {\left[\left.\frac{\partial}{\partial \varepsilon} \operatorname{Rm}(g+\varepsilon h)\right|_{\varepsilon=0}\right]_{i j k}^{l} } \\
= & \frac{1}{2} g^{l p}\left(\nabla_{i} \nabla_{j} h_{k p}+\nabla_{i} \nabla_{k} h_{j p}-\nabla_{i} \nabla_{p} h_{j k}\right. \\
& \left.\quad-\nabla_{j} \nabla_{i} h_{k p}-\nabla_{j} \nabla_{k} h_{i p}+\nabla_{j} \nabla_{p} h_{i k}\right)+ \text { LOT. }
\end{aligned}
$$

Here, we use $g$ to denote the metric about which we are linearizing and we use $h$ to denote the tangent to the linearization; we note that covariant derivatives and curvature terms appearing here and below are calculated with respect to $g$, and indices are raised and lowered using $g$. The term LOT denotes lower order terms with respect to derivatives of $h$. Applying (3) together with the observations that $\left[D g^{-1}(h)\right]^{i j}=-h^{i j}$ and $\nabla_{p} \nabla_{j} h_{n}^{l}=\nabla_{j} \nabla_{p} h_{n}^{l}-R_{j p n}^{m} h_{m}^{l}+R_{j p m}^{l} h_{n}^{m}$, we calculate

$$
\begin{aligned}
{\left[D \mathrm{Rm}_{g}^{2}(h)\right]_{i j}=\frac{1}{2} } & g^{p k} R_{i k l}^{n}\left(\nabla_{j} \nabla_{p} h_{n}^{l}+\nabla_{j} \nabla_{n} h_{p}^{l}-\nabla_{j} \nabla^{l} h_{p n}\right. \\
& \left.-\nabla_{p} \nabla_{j} h_{n}^{l}-\nabla_{p} \nabla_{n} h_{j}^{l}+\nabla_{p} \nabla^{l} h_{j n}\right) \\
& +\frac{1}{2} g^{p k} R_{j p n}^{l}\left(\nabla_{i} \nabla_{k} h_{l}^{n}+\nabla_{i} \nabla_{l} h_{k}^{n}-\nabla_{i} \nabla^{n} h_{k l}\right. \\
& \left.-\nabla_{k} \nabla_{i} h_{l}^{n}-\nabla_{k} \nabla_{l} h_{i}^{n}+\nabla_{k} \nabla^{n} h_{i l}\right)+ \text { LOT } \\
= & R_{i k l u}\left(\nabla_{j} \nabla^{l} h^{k u}-\nabla^{k} \nabla^{l} h_{j}^{u}\right)+R_{j k l u}\left(\nabla_{i} \nabla^{l} h^{k u}-\nabla^{k} \nabla^{l} h_{i}^{u}\right)+\text { LOT. }
\end{aligned}
$$

We obtain the principal symbol of the full flow (2) by replacing each $\nabla$ appearing in the sum of the Laplacian plus the terms in (4) by the co-vector $\xi$. As in the Ricci flow case (see [9]), we work in orthonormal coordinates, and without loss of 
generality, we assume that $\xi_{1}=1, \xi_{l}=0, l \geq 2$. Writing the derivative of the right hand side of (2) as $D L_{g}$, the symbol is thus given by

$$
\begin{aligned}
& \sigma D L_{g}(h)_{i j}:=\sigma D L_{g}(\xi)(h)_{i j}=h_{i j} \\
& \quad+\frac{\alpha}{2} R_{i k 1 u} \delta_{j 1} h^{k u}-\frac{\alpha}{2} R_{i 11 u} h_{j}^{u}+\frac{\alpha}{2} R_{j k 1 u} \delta_{i 1} h^{k u}-\frac{\alpha}{2} R_{j 11 u} h_{i}^{u} .
\end{aligned}
$$

Noting that (by design) the left hand side of (5) takes the form of a linear algebraic operator on $h$, we rewrite the right hand side of (5) formally as a matrix expression,

$$
\sigma D L_{g}(h)_{A}=\Sigma_{A}^{B} h_{A} .
$$

The capital Latin letters represent the symmetric indices on $h$ :

$$
h_{A} \leftrightarrow\left\{h_{11}, h_{12}, \ldots, h_{1 n}, h_{22}, h_{23}, \ldots, h_{2 n}, \ldots, h_{n n}\right\},
$$

with, for example, $h_{21}$ not appearing since $h_{12}$ does. We note that $h_{A}$ is an $n(n+1) / 2$ dimensional vector, and correspondingly $\Sigma_{A}^{B}$ is an $n(n+1) / 2 \times$ $n(n+1) / 2$ matrix, whose columns are given by the $h_{B}$ terms of $\sigma D L_{g}(h)_{A}$.

To determine whether or not the PDE system (2) is parabolic for a given metric $g$ (representing the initial data for an RG-2 flow solution) one determines if the matrix $\Sigma_{A}^{B}$ is nondegenerate for that metric. We verify here that the condition stated for sectional curvatures in the hypothesis of Theorem 1 guarantees this nondegeneracy, and consequently the parabolicity of (2). To carry out this verification explicitly, it is useful to write out the components of equation (5) for various possible choices of the indices $i j$. There are three cases to consider:

Case 1. $i=j=1$ :

$$
\sigma D L_{g}(h)_{11}=h_{11}+\alpha R_{1 k 1 u} h^{k u}
$$

Case 2. $i=1, j \neq 1$ :

$$
\sigma D L_{g}(h)_{1 j}=h_{1 j}+\frac{\alpha}{2} R_{j k 1 u} h^{k u}-\frac{\alpha}{2} R_{j 11 u} h_{1}^{u},
$$

Case 3. $i \neq 1, j \neq 1$ :

$$
\sigma D L_{g}(h)_{i j}=h_{i j}+\frac{\alpha}{2} R_{1 i 1 u} h_{j}^{u}+\frac{\alpha}{2} R_{1 j 1 u} h_{i}^{u}
$$

Based on these expressions, one sees that the matrix $\Sigma_{A}^{B}$ takes the block form

$$
\Sigma_{A}^{B}=\left(\begin{array}{ll}
I & \lambda \\
\mu & \nu
\end{array}\right)
$$

Here $I$ is the $n \times n$ identity matrix, and since $n(n+1) / 2-n=n(n-1) / 2$, the block $\mu$ is an $n \times n(n-1) / 2$ matrix composed entirely of zeros. Thus the determinant of $\Sigma_{A}^{B}$ is given by $\operatorname{det}(\nu)$, from which it follows that the system (2) is parabolic so long as the $n(n-1) / 2 \times n(n-1) / 2$ matrix $\nu$ has nonvanishing determinant. One determines the components of $\nu$ from expression (10). The diagonal components of $\nu$ have $A=B=i j$ (so they are the $h_{i j}$ terms of $\sigma D L_{g}(h)_{i j}$ for $i, j>n$ ), and have the form

$$
1+\frac{\alpha}{2} R_{1 i 1 i}+\frac{\alpha}{2} R_{1 j 1 j}
$$

These terms thus take the form $1+\alpha K_{A}$, for sectional curvatures $K_{A}$.

By contrast, the off-diagonal terms of $\nu$ do not involve the sectional curvatures; rather, they involve only mixed curvatures of the form $R_{1 i 1 u}$ and $R_{1 j 1 u}$ for $u \neq i$ and $u \neq j$. Standard arguments show that we can choose an orthonormal basis which 
diagonalizes the (symmetric) matrix $R_{1 m 1 n}$; it follows that $\nu$ is nondegenerate so long as the condition $1+\alpha K_{A}>0$ holds, thus proving our theorem.

Example 1 (4-dimensions). To help clarify the discussion in the proof above, we write out the matrix $\nu$ explicitly for $\mathrm{n}=4$ dimensions. The order of the columns here is $h_{22}, h_{23}, h_{24}, h_{33}, h_{34}, h_{44}$. Thus, for example, the first row in this matrix is obtained by writing out $\sigma D L_{g}(h)_{22}=\left(1+\alpha R_{1212}\right) h_{22}+\alpha R_{1213} h_{23}+\alpha R_{1214} h_{24}$. The complete set of entries are as follows:

$$
\nu=\left(\begin{array}{ccc}
1+\alpha R_{1212} & \alpha R_{1213} & \alpha R_{1214} \\
\frac{\alpha}{2} R_{1312} & 1+\frac{\alpha}{2}\left(R_{1313}+R_{1212}\right) & \frac{\alpha}{2} R_{1314} \\
\frac{\alpha}{2} R_{1214} & \frac{\alpha}{2} R_{1314} & 1+\frac{\alpha}{2}\left(R_{1212}+R_{1414}\right) \\
0 & \alpha R_{1213} & 0 \\
0 & \frac{\alpha}{2} R_{1214} & \frac{\alpha}{2} R_{1213} \\
0 & 0 & \alpha R_{1214}
\end{array}\right.
$$

$\left.\begin{array}{ccc}0 & 0 & 0 \\ \frac{\alpha}{2} R_{1213} & \frac{\alpha}{2} R_{1214} & 0 \\ 0 & \frac{\alpha}{2} R_{1213} & \frac{\alpha}{2} R_{1214} \\ 1+\alpha R_{1313} & \alpha R_{1314} & 0 \\ \frac{\alpha}{2} R_{1314} & 1+\frac{\alpha}{2}\left(R_{1313}+R_{1414}\right) & \frac{\alpha}{2} R_{1314} \\ 0 & \alpha R_{1314} & 1+\alpha R_{1414}\end{array}\right)$

We can diagonalize $R_{1 m 1 n}$, ensuring that the off-diagonal terms are zero.

\section{REFERENCES}

[1] John A. Buckland, Short-time existence of solutions to the cross curvature flow on 3-manifolds, Proc. Amer. Math. Soc. 134 (2006), no. 6, 1803-1807 (electronic), DOI 10.1090/S0002-9939-05-08204-3. MR2207496 (2007a:35061)

[2] Bennett Chow and Dan Knopf, The Ricci flow: an introduction, Mathematical Surveys and Monographs, vol. 110, American Mathematical Society, Providence, RI, 2004. MR2061425 (2005e:53101)

[3] L. Cremaschi and C. Mantegazza, Short-time Existence of the second order Renormalization Group flow in dimension three. arXiv:1306.1721

[4] Dennis M. DeTurck, Deforming metrics in the direction of their Ricci tensors, J. Differential Geom. 18 (1983), no. 1, 157-162. MR697987 (85j:53050)

[5] Christine Guenther, James Isenberg, and Dan Knopf, Stability of the Ricci flow at Ricci-flat metrics, Comm. Anal. Geom. 10 (2002), no. 4, 741-777. MR1925501 (2003g:53118)

[6] Karsten Gimre, Christine Guenther, and James Isenberg, Second-order renormalization group flow of three-dimensional homogeneous geometries, Comm. Anal. Geom. 21 (2013), no. 2, 435-467, DOI 10.4310/CAG.2013.v21.n2.a7. MR.3043753

[7] K. Gimre, C. Guenther, and J. Isenberg, A geometric introduction to the 2-loop renormalization group flow. To appear: Festschrift Volume in honor of Mme Choquet-Bruhat, J.Fixed Pt. Theory Appl.

[8] Christine Guenther and Todd A. Oliynyk, Stability of the (two-loop) renormalization group flow for nonlinear sigma models, Lett. Math. Phys. 84 (2008), no. 2-3, 149-157, DOI 10.1007/s11005-008-0245-8. MR2415546 (2009c:81060)

[9] Richard S. Hamilton, Three-manifolds with positive Ricci curvature, J. Differential Geom. 17 (1982), no. 2, 255-306. MR664497 (84a:53050) 
[10] Todd A. Oliynyk, The second-order renormalization group flow for nonlinear sigma models in two dimensions, Classical Quantum Gravity 26 (2009), no. 10, 105020, 8, DOI 10.1088/02649381/26/10/105020. MR2532057 (2010g:81173)

Department of Mathematics, Columbia University, New York City, New York 10027

E-mail address: gimre@math.columbia.edu

Department of Mathematics and Computer Science, Pacific University, Forest Grove, Oregon 97116

E-mail address: guenther@pacificu.edu

Department of Mathematics, University of Oregon, Eugene, Oregon 97403

E-mail address: isenberg@uoregon.edu 